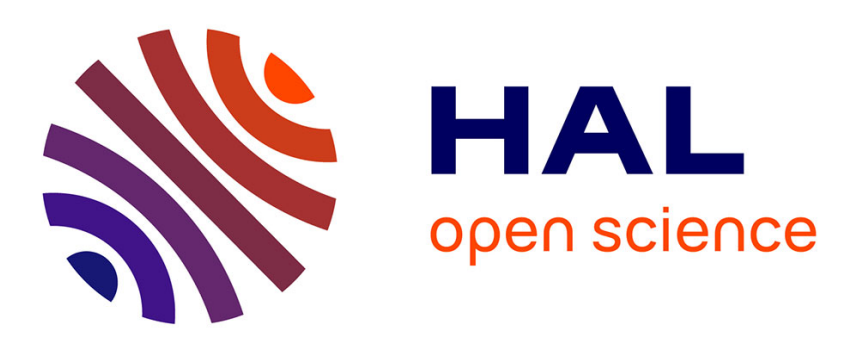

\title{
Adherence control for electric vehicles on varying road conditions
}

\author{
Marcel Stefan Geamanu, Hugues Mounier, Silviu-Iulian Niculescu, Arben \\ Cela, Guenael Le Solliec
}

\section{- To cite this version:}

Marcel Stefan Geamanu, Hugues Mounier, Silviu-Iulian Niculescu, Arben Cela, Guenael Le Solliec. Adherence control for electric vehicles on varying road conditions. 21st Mediterranean Conference on Control and Automation (MED), Jun 2013, Platanias, Greece. 10.1109/MED.2013.6608904 . hal02194639

\section{HAL Id: hal-02194639 \\ https://hal.science/hal-02194639}

Submitted on 16 Dec 2019

HAL is a multi-disciplinary open access archive for the deposit and dissemination of scientific research documents, whether they are published or not. The documents may come from teaching and research institutions in France or abroad, or from public or private research centers.
L'archive ouverte pluridisciplinaire HAL, est destinée au dépôt et à la diffusion de documents scientifiques de niveau recherche, publiés ou non, émanant des établissements d'enseignement et de recherche français ou étrangers, des laboratoires publics ou privés. 


\section{Adherence control for electric vehicles on varying road conditions}

\author{
Marcel Stefan Geamanu, \\ Hugues Mounier and \\ Silviu-Iulian Niculescu \\ Laboratoire des Signaux et Systèmes (L2S) \\ UMR 8506 CNRS-Supelec, \\ 3 rue Joliot Curie, 91192 Gif-sur-Yvette, FRANCE
}

\author{
Arben Cela \\ UPE ESIEE Paris, \\ 2 boulevard Blaise Pascal, \\ Guénaël LeSolliec \\ and Marcel Stefan Geamanu \\ IFPEN, 1 et 4 avenue de Bois-Préau, \\ 93162 Noisy le Grand, FRANCE 92500 Rueil-Malmaison, FRANCE
}

\begin{abstract}
The present paper describes a torque saturation control technique applied on vehicular control, operating on time-varying tire-road adherence conditions and with noise perturbation. The method is based on an instantaneous estimation of the maximum available friction using the Dugoff tire model [3]. The novelty lies in the modeling of the road conditions, which are regarded as continuous variables. A "dynamic" Pacejka model is built around the classical Pacejka model, giving a more realistic approach of the tire-road interaction. The implemented estimation method has to adapt to all the parameter changes, to produce a reliable maximum friction on which the control will be applied. The complex modeling of the road conditions will be enlarged with a noise perturbation, to test our method's robustness, which represents the objective of the present work. At the same time, the vehicle is considered to be equipped with "in-wheel" electrical motors, which provide a quick transmission of the torque directly at the wheel.
\end{abstract}

\section{INTRODUCTION}

In vehicular safety, two embedded systems are crucial in good behavior of the vehicle. The first one is the ABS (Antiblockier System) which prevents the wheel from blocking in case of hard braking maneuver as described in [2], [4], [13]. The second one is the TCS (Traction Control System) which prevents the wheel from spinning in case of hard accelerating maneuver [11]. Both of these systems are based on the friction between the tire and the road and depending on it, they act on the appropriate sub-systems of the vehicle which act on the brakes or the acceleration respectively. Hence, the tire-road friction plays an important role in the good functioning of ABS and TCS and so, a good estimation of the friction is needed. Nevertheless, the influence of the friction on the longitudinal dynamics is hard to quantify since it depends on numerous factors which are not easily separable.

For a better understanding of this phenomena it is helpful to look closer at the Pacejka formula [14], which is an empirical formula whose results are usually close to the reality. Nevertheless, the curves remain at theoretical level,

Emails: marcel-stefan.geamanu@1ss.supelec.fr (corresponding author), hugues.mounier@1ss.supelec.fr, silviu.niculescu@1ss.supelec.fr, a.cela@esiee.fr, guenael.le-solliec@ifpen.fr, since a slight change in the parameters yield different shapes of the curves. Instead of the curves modeled by Pacejka, in real environments we find a cloud of points whose position can vary from a sample time to another for the same road conditions [8], [15], as shown in figure 1. In addition, perturbations and noise can easily affect the estimation process with possible influence on the final control applied at the wheels.

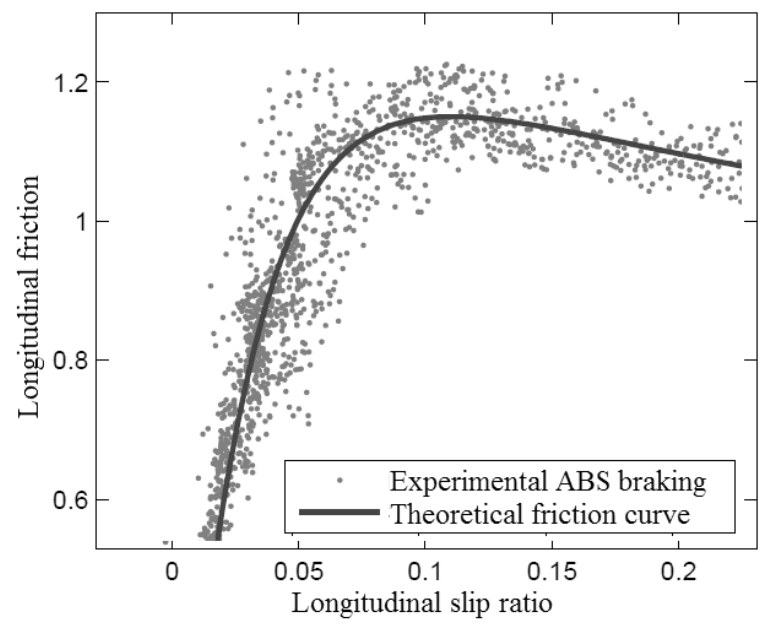

Fig. 1: Experimental friction estimation

In this paper, a different approach regarding the Pacejka model is presented. Here, instead of considering only three theoretical curves modeling the main types of road surfaces (dry, wet and snowy), we interpret them as continuously varying during the driving maneuver. This approach will give a more realistic modeling of the road surface conditions and will allow to have a better view of the results of the proposed method in this environment. At the same time, adding noise on the measurement variables will bring even more realism to the model, allowing in addition to test the robustness of our method. Another innovation of the present work consists in using the in-wheel electric motor as the only actuator in acceleration and deceleration, in order to provide the 
necessary torque to accomplish both TCS and ABS functions. The in-wheel motor used here is powerful $(39 \mathrm{~kW})$, it has a very low latency and is able to provide a braking torque on the wheels [10] faster than conventional brakes which are normally used for this purpose. With the electric motor, due to a good knowledge of its output torque computed from the current intensity that passes through the motor, one can envisage the estimation of the tire-road friction forces. Unlike the existing, rather conservative, control strategies, described in [2], [4], [5], [9], [12], which rely on the longitudinal slip and on a fixed threshold, the electric motor allows to apply a control on the friction coefficient, while considering the road conditions.

The remaining of the paper is organized as follows: in section 2, a new approach into modeling the road conditions is presented; in section 3, the vehicle model, the estimation method and the control strategy are described; section 4 shows the results of the proposed control method; some concluding remarks are presented in section 5 .

\section{CONTINUOUS VARIATION OF THE ROAD CONDITIONS}

Classical Pacejka formula is expressed as follows [14]:

$$
F_{x}=D \sin (C \arctan (B \lambda-E(B \lambda-\arctan (B \lambda))))
$$

with $F_{x}$ being the longitudinal force and $\lambda$ the longitudinal slip ratio. The $B, C, D$ and $E$ parameters are calculated as follows:

- $C=b_{0}$

- $D=\left(b_{1} F_{z}+b_{2}\right) F_{z}$

- $B=\left(\left(b_{3} F_{z}^{2}+b_{4} F_{z}\right) e^{-b_{5} F_{z}}\right) / C D$

- $E=b_{6} F_{z}^{2}+b_{7} F_{z}+b_{8}$

with $F_{z}$ being the normal force on the tire. The constant parameters $b_{0}-b_{8}$ have fixed values depending on the type of the utilized tire. Here, $b_{0}=1.5699, b_{1}=-25.63, b_{2}=$ $1305, b_{3}=6.825, b_{4}=395.69, b_{5}=0, b_{6}=0.0034, b_{7}=$ $-0.0082, b_{8}=0.6565$. In this formula, parameters $C$ and $D$ have the most noticeable influence on the curves. One interpretation of the Pacejka coefficients is the following:

- $C$ represents the behavior of the curves once the maximum value is exceeded. A small $C$ will be translated in a small slope of the curve after its peak. This parameter has also an influence on the slope of the pseudo-linear segment of the curves.

- $D$ is the maximum force the tire can generate, at its peak performance, influencing also the slope of the curves as shown in figure 3.

The effect of parameters $C$ and $D$ on the friction curves is shown in figure 2 and 3 . So, instead of using fixed parameters in the computation of $C$ and $D$, we can consider them as time-varying in order to model the variation of road conditions in real situations. In these situations estimation can be problematic, as the road conditions change continuously. To model this variation, we have summed up parameters $C$ and $D$ into one single variable which gives the state of the road, called $X_{r}$. Modeling $C$ and $D$ into one single variable represents the realistic case of the friction variation.

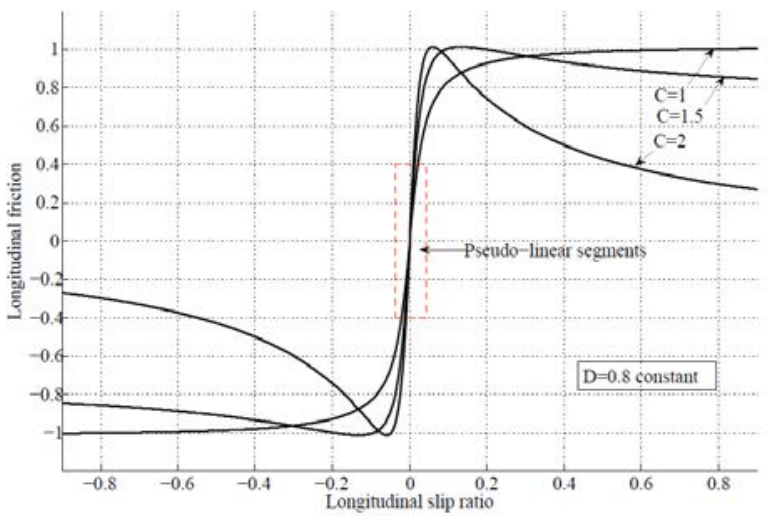

Fig. 2: Parameter $C$ influence on the friction curves

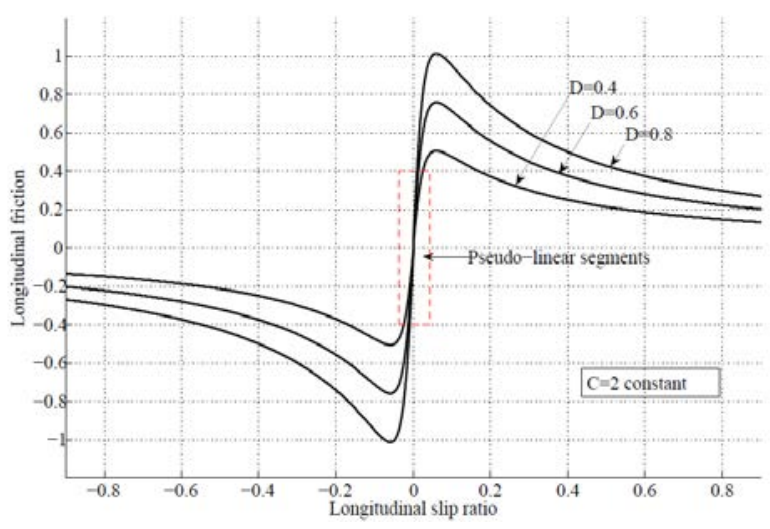

Fig. 3: Parameter $D$ influence on the friction curves

The friction between the wheels and the road is subject to variation, its values changing due to numerous factors as the weather conditions (hot temperatures, rain, snow or ice), road maintenance and type of the pavement (asphalt, concrete or cobblestone). Therefore, $X_{r}$ will vary between [0..1], giving a maximum adherence when is close to value 1 (simulating a dry asphalt road for example), and a small adherence when is close to value 0 (simulating a snowy or icy road), but will take into account all the other adherences in between, modeled as a continuous variation of $C$ and $D$. Parameters $C$ and $D$ will have the following expressions:

$$
\begin{aligned}
& C=X_{r}+k_{c} \\
& D=\frac{k_{d_{1}}}{X_{r}+k_{d_{2}}}+X_{r} k_{d_{3}}
\end{aligned}
$$

with $k_{c}, k_{d_{1}}, k_{d_{2}}, k_{d_{3}}$ being design parameters. Therefore, Pacejka formula will be rewritten as follows:

$$
\begin{array}{r}
F_{x}=\left(\frac{k_{d_{1}}}{X_{r}+k_{d_{2}}}+X_{r} k_{d_{3}}\right) \sin \left(\left(X_{r}+k_{c}\right) \arctan (B \lambda-\right. \\
E(B \lambda-\arctan (B \lambda))))
\end{array}
$$

Therefore, we have the variable $X_{r}$ which will give us the state of the road surface and we can use this input variable to model a continuous variation of the road surface condition. This will yield a more realistic approach of 
Pacejka curves, regarded as multiple time-varying curves, as shown in figure 4 . Here we pass through snowy roads with $\mu_{x_{\max }}=0.4 \sim 0.5$ and rainy roads with $\mu_{x_{\max }}=0.7 \sim 0.8$ towards dry roads with $\mu_{x_{\max }}=0.9 \sim 1$. Nevertheless, the curves between these values are also taken into account, giving an approach to model road conditions closer to reality. To model the continuous change of Pacejka parameters, in

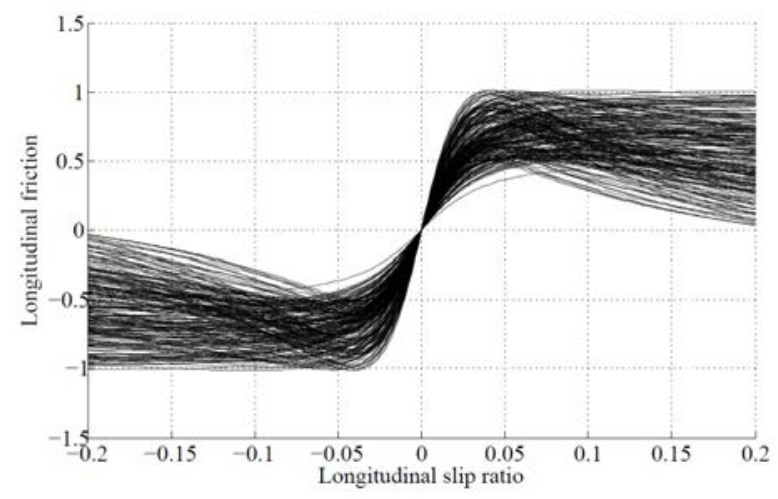

Fig. 4: Realistic modeling of Pacejka curves

the simulation we used the following time evolution of the variable $X_{r}$ arbitrary chosen, which gives us the state of the road surface, as shown in figure 5: This profile passes from

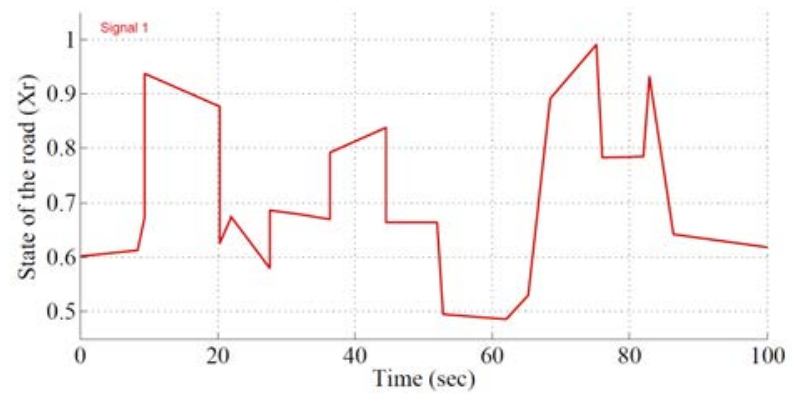

Fig. 5: Time evolution of the state of the road

dry road surface (simulation time $70 \mathrm{~s}-80 \mathrm{~s}$ ), through rainy road surface (simulation time 10s-20s, 35s-45s) and snowy road surface (simulation time 54s-65s). In this way, we model variation of the parameters via a more realistic approach, testing the performances of our Dugoff-based maximum friction estimation.

\section{VEHICLE DYNAMICS AND CONTROL}

\section{A. Vehicle model}

To test our estimation method along with the control applied on it, we have chosen a simple vehicle model as shown in [7]. Even though it is a simple model, it will allow us to test the efficiency and robustness of our approach. The equations of the dynamics of the vehicle and of the wheel can be written as follows:

$$
\begin{aligned}
m \dot{V}_{x} & =F_{x}-F_{\text {aero }}, \\
I \dot{\omega} & =T-r F_{x}-R_{x}, \\
F_{x} & =\mu_{x} F_{z}, \\
\lambda & =\frac{r \omega-V_{x}}{\max \left(V_{x}, r \omega\right)},
\end{aligned}
$$

where: $m$ is the quarter vehicle mass $(\mathrm{kg}), V_{x}$ is the chassis speed $(\mathrm{m} / \mathrm{s}), F_{z}=m g$ is the normal force on the tire $(\mathrm{N})$, $F_{\text {aero }}=\left(\rho C_{a} A V_{x}^{2}\right) / 2$ is the aerodynamic drag force $(\mathrm{N})$, $R_{x}=m g C_{r}$ is the rolling resistance force $(\mathrm{N}), F_{x}$ is the longitudinal force $(\mathrm{N}), T$ is the driving/braking torque $(\mathrm{Nm})$, $\lambda$ is the longitudinal slip ratio, $\omega$ is the wheel velocity ( $\mathrm{rad} / \mathrm{s}$ ) and $r$ is the effective tire radius (m).

In the model we have considered a Pacejka modeling of the friction, as presented in section 2, which will give an approach closer to a realistic tire-road friction environment. Driver's actions (acceleration or braking) can be translated in different torque inputs, depending of the driver requirements. The driver model is described in [6].

Once the driver torque is computed, it will have to be limited as function of the maximum available friction, in order to avoid wheel slip in acceleration or wheel skid in braking maneuver.

\section{B. Maximum friction estimation using Dugoff tire-model [6]}

Dugoff tire model [3] has an interesting feature, assuming a uniform vertical pressure distribution on the tire contact patch. This is a simplification compared to the more realistic parabolic pressure assumed in Pacejka model (figure 6). However, the longitudinal forces are directly related to the maximum friction coefficient in more simple equations than in Pacejka model, hence the interest to estimate Dugoff parameters in order to obtain a maximum friction coefficient estimation.

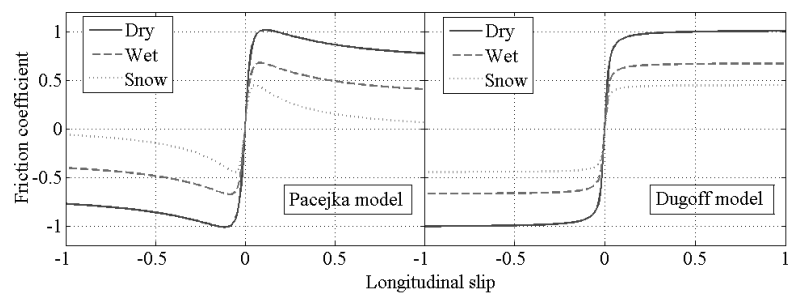

Fig. 6: Friction coefficients compared on Pacejka and Dugoff curves.

In Dugoff's tire-model, longitudinal efforts are modeled as follows:

$$
F_{x}^{D}=f(\tau) K_{x} \lambda .
$$

where $f(\tau)$ is a piecewise function:

$$
f(\tau)=\left\{\begin{array}{ll}
(2-\tau) \tau, & \tau<1 \\
1, & \tau \geqslant 1
\end{array}, \quad \tau=\frac{\mu_{x_{\max } F_{z}}}{2\left|K_{x} \lambda\right|}\right.
$$

It is not difficult to see that $\mu_{x_{\max }}$ can be expressed in terms of four a priori known variables $F_{x}, F_{z}, \lambda, K_{x}$. 
The $\mu-\lambda$ characteristics have two specific regions. The first one is linear corresponding to a single value $\tau=1$. The second is nonlinear defined for all values of $\tau$ inferior to 1 . In the linear region, the longitudinal efforts are calculated as $F_{x}^{D}=K_{x} \lambda$. Therefore, the values for $K_{x}$ can be derived in the linear region of the friction curves $(f(\tau)=1)$, as shown in [6].

Next, let us take the non linear region case of the $f(\tau)$ function, i.e. $f(\tau)=(2-\tau) \tau$. Then, the longitudinal efforts can be expressed as follows:

$$
F_{x}^{D}=\left(2-\frac{\mu_{x_{\max }} F_{z}}{2\left|K_{x} \lambda\right|}\right) \frac{\mu_{x_{\max }} F_{z}}{2\left|K_{x} \lambda\right|} K_{x} \lambda .
$$

This expression can be rewritten as a second algebraic equation of the maximum friction coefficient:

$$
\mu_{x_{\max }}^{2} F_{z}^{2}-4 \mu_{x_{\max }}\left|K_{x} \lambda\right| F_{z}+4\left|K_{x} \lambda\right| F_{x}^{D}=0,
$$

whose two solutions are:

$$
\mu_{x_{\max }}=\frac{2\left(\left|K_{x} \lambda\right| \pm \sqrt{K_{x} \lambda\left(K_{x} \lambda-F_{x}^{D}\right)}\right)}{F_{z}} .
$$

As observed in "off-line" calculations, Dugoff tire model saturates at a different peak value than Pacejka tire model. It is in fact a weighting factor (called $\alpha$ ) between Dugoff and Pacejka models that drives Dugoff model to cross through Pacejka model exactly in the peak of the curve (figure 7). Its values can be calculated only close to the peak of the $\mu-\lambda$ curve. The two key parameters, $K_{x}$ and $\alpha$ are used in the computation of the maximum friction coefficient and their estimation is presented in [6]. Hence, we are using a simple Dugoff tire model in order to achieve an on-line estimation of a more complex Pacejka tire model which is closer to a realistic friction environment.

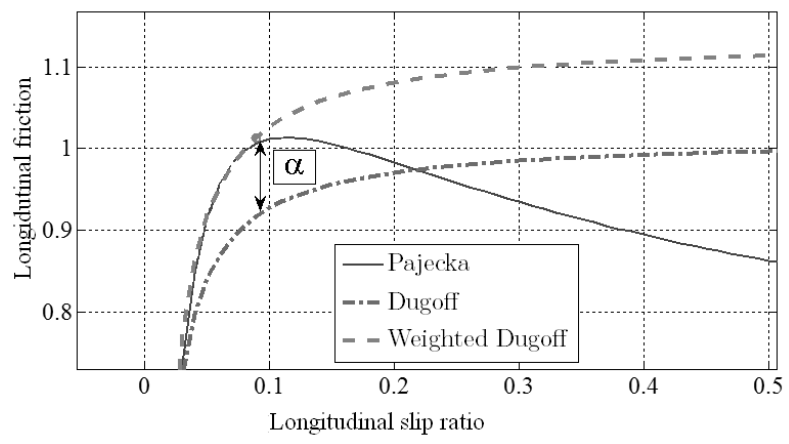

Fig. 7: Weighting parameter $\alpha$ at the peak of longitudinal efforts built with Pacejka and Dugoff models.

\section{Torque saturation control}

A high value of the torque computed from driver's actions will induce wheel slip in acceleration or wheel skid in braking maneuver. Therefore, its value has to be limited with a computed torque that takes into account the state of the road and its maximum available friction. The proposed control method will saturate the demanded torque, coming from driver's requirements, with a maximum torque value, which is computed starting from wheel dynamics Eq.(6) of the overall model. Replacing $F_{x}$ by Eq.(7), and extracting the torque $T$ will yield:

$$
T=I \dot{\omega}+r \mu_{x} F_{z}+R_{x} .
$$

Therefore, the saturation torque will be given by:

$$
T_{\text {sat }}=I \dot{\omega}+r \mu_{x_{\max }} F_{z}+R_{x} .
$$

The maximum friction coefficient $\mu_{x_{\max }}$ will be calculated following the estimation strategy presented in subsection B.

\section{Simulation Results}

\section{A. Noise-free environment}

Along with the state of the road profile input shown in figure 5 , the following speed profile was used in simulation. In figure 8 we find hard acceleration and braking phases, simulated to push the estimation and control strategies at their limits and to test the robustness of the proposed method.

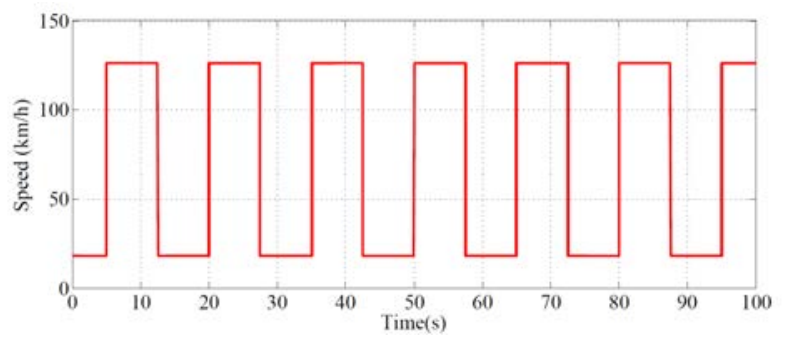

Fig. 8: Speed profile used in the simulations

Having set up the estimation of the maximum friction estimation as described in section 2.B, along with the control strategy presented in section 2.C, will yield the following result in terms of maximum friction tracking.

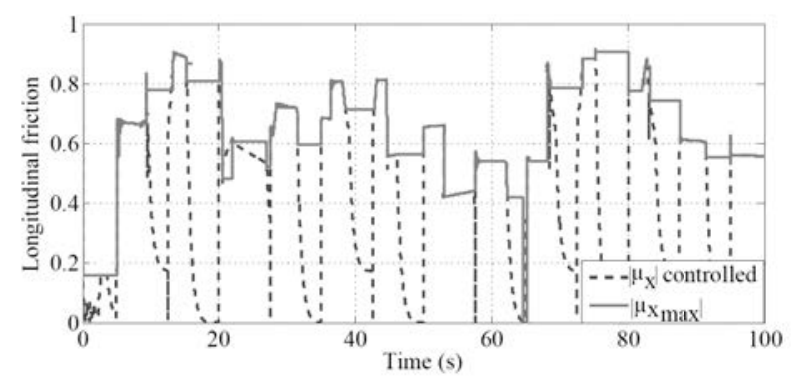

Fig. 9: Maximum friction tracking

In figure 9 can be seen that even though the maximum friction changes in time, the estimation method provides a reliable value for $\mu_{x_{\max }}$. The $\mu_{x_{\text {controlled }}}$ line in figure 9 shows that the instantaneous friction never exceeds the maximum estimated value, therefore accomplishing the purpose of the control. An interesting fact happens at simulation time $\mathrm{t}=53 \mathrm{~s}$. Here the maximum friction drops from approximately 0.7 to 0.4 during the acceleration phase. In other words we go from a rainy road to a snowy road. Nevertheless, the control tracks this variation of the maximum available friction, giving a stable wheel behavior, as shown in figure 
10.b. Given the variation of parameter $X_{r}$ which will also induce the variation of parameters $C$ and $D$ of Pacejka formula, the slope of the linear segment of the friction curves is continuously changing. In our strategy, the slope of the linear segment is defined by parameter $K_{x}$. We have computed its off-line values for the state of the road profile shown in figure 5 to have a reference value to compare with its estimated value. Its evolution is shown in figure 11 and one can see that even if the slope varies continuously, the estimation follows the modeled value. Also, one has to take into account that $K_{x}$ updates only when the values of the longitudinal slip are in the linear zone of the friction characteristics.
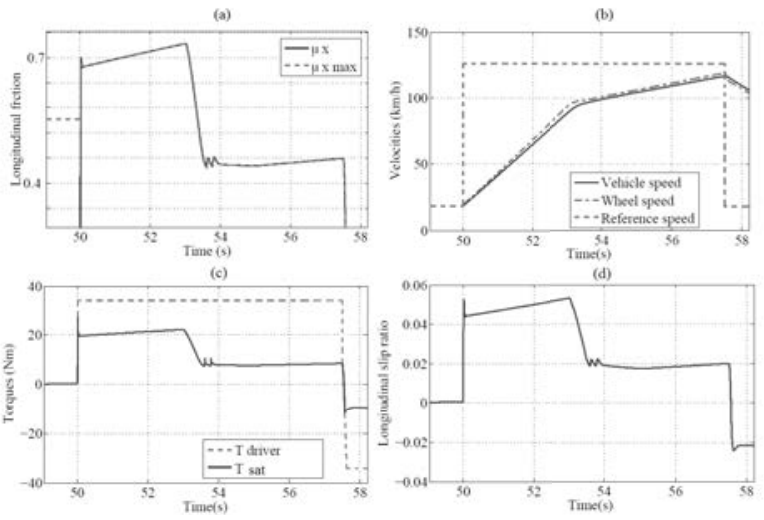

Fig. 10: Maximum friction variation tracking

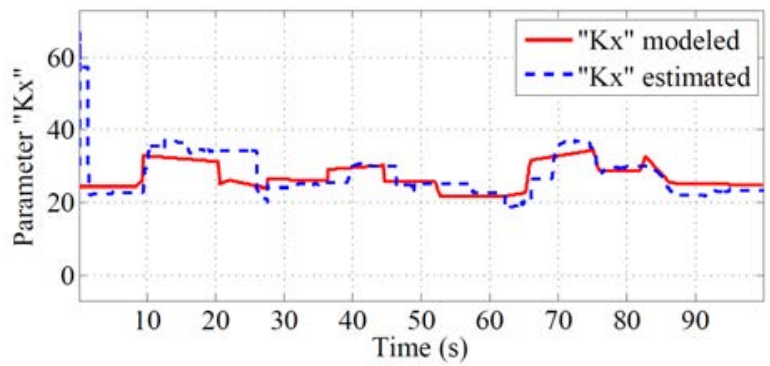

Fig. 11: Reference and estimated value for $K_{x}$

The adaptation parameter $\alpha$ will also be variable, depending on the state of the road. As in the case of $K_{x}$, off-line values for $\alpha$ were computed, for the same state of the road input $X_{r}$. Its evolution is shown in figure 12. The range of variation of $\alpha$ is reduced compared to the one of $K_{x}$. The estimation of parameter $\alpha$ will compensate the errors that arise in the estimation of $K_{x}$, therefore its estimated values differ from the the modeled values, yet they follow the modeled profile. The large variation of Pacejka parameters brings a modeling closer to real situations, giving estimations that no longer stay only one curve, but on multiple curves, as shown in figure 13 .

Even if the estimation points seem to be more dispersed than in a conventional modeling, it can be seen in figure 13 that $\mu(\lambda)$ never exceeds the peak of the curves, showing good performance of the control scheme. The conditions vary

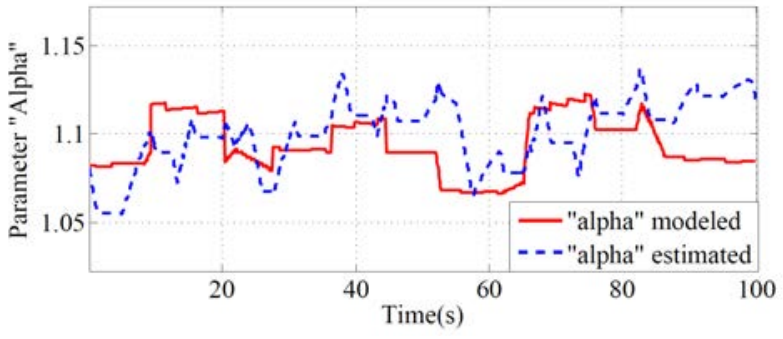

Fig. 12: Reference and estimated value for $\alpha$

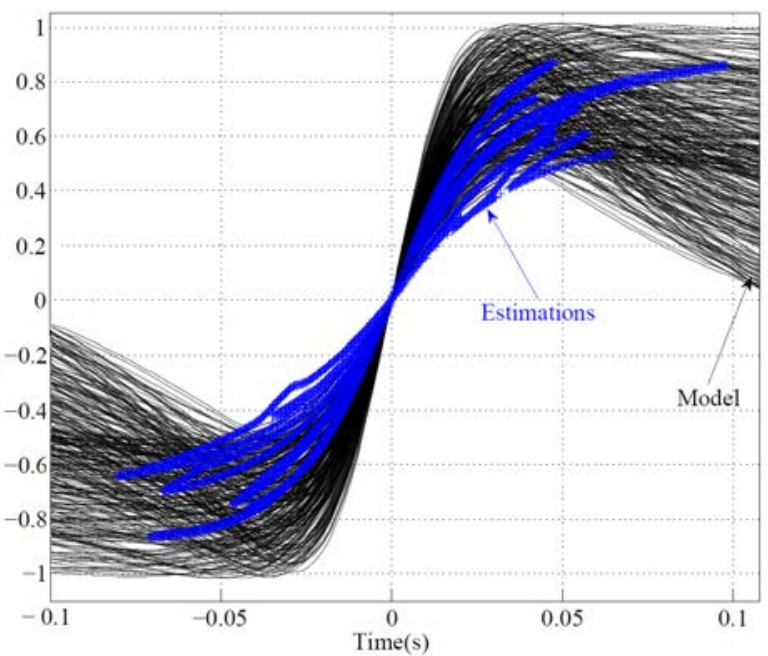

Fig. 13: Friction estimation on time-varying road conditions

from rainy roads with $\mu_{x_{\max }}=0.9$ to snowy roads with $\mu_{x_{\max }}=0.3$. So, a large range of tire-road friction is ran through, testing the estimation and control methods and their limits, yielding good results in terms of friction tracking and vehicle behavior.

\section{B. Noise perturbation}

In real systems, noise can affect the performances of the estimation strategy propagating to the control that is applied at the wheel. In the following we take into account a random noise coming from wheel acceleration that affects the estimation of $\mu_{x_{\max }}$.

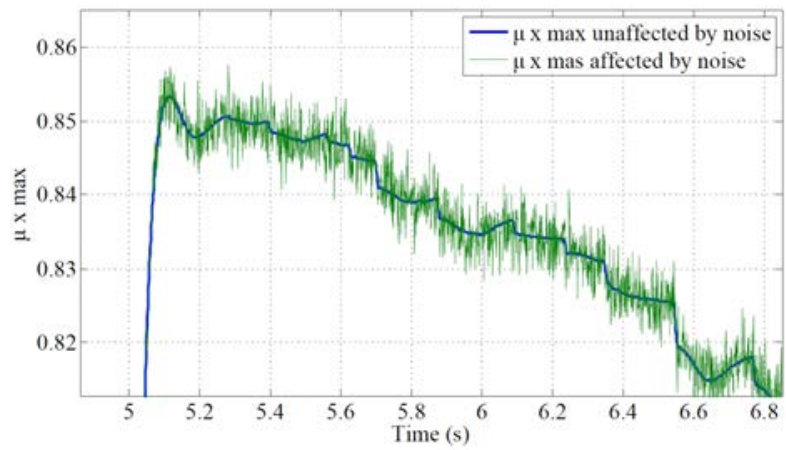

Fig. 14: Noise affecting the estimation of $\mu_{x_{\max }}$

Adding noise will increase the complexity of the problem, 
since it can propagate at the final control applied at the wheels. As seen in figure 15, the noise coming from the maximum friction estimation is propagated on the computation of the control. But, having taken into account the filtering provided by the electric motors, with their small delays, the final torque applied at the wheels is less affected by the noise (see figure 15).

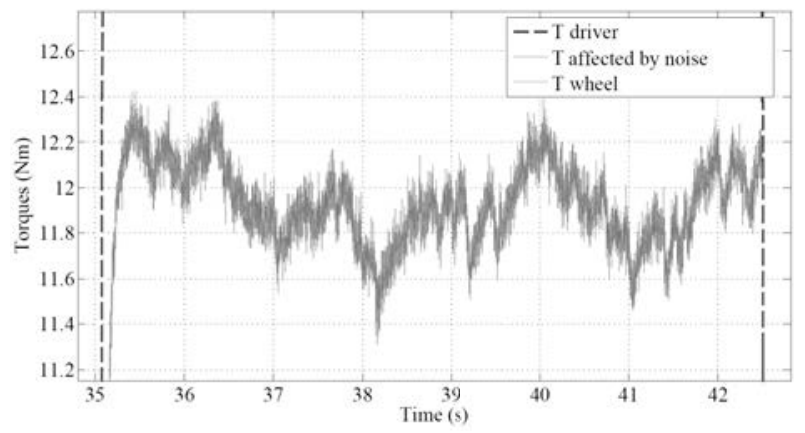

Fig. 15: Noise affecting the computation of $T_{\text {sat }}$

Hence, even in noisy environment with continuous variation of Pacejka parameters, the control performs good tracking of the maximum friction, as seen in figure 16 .
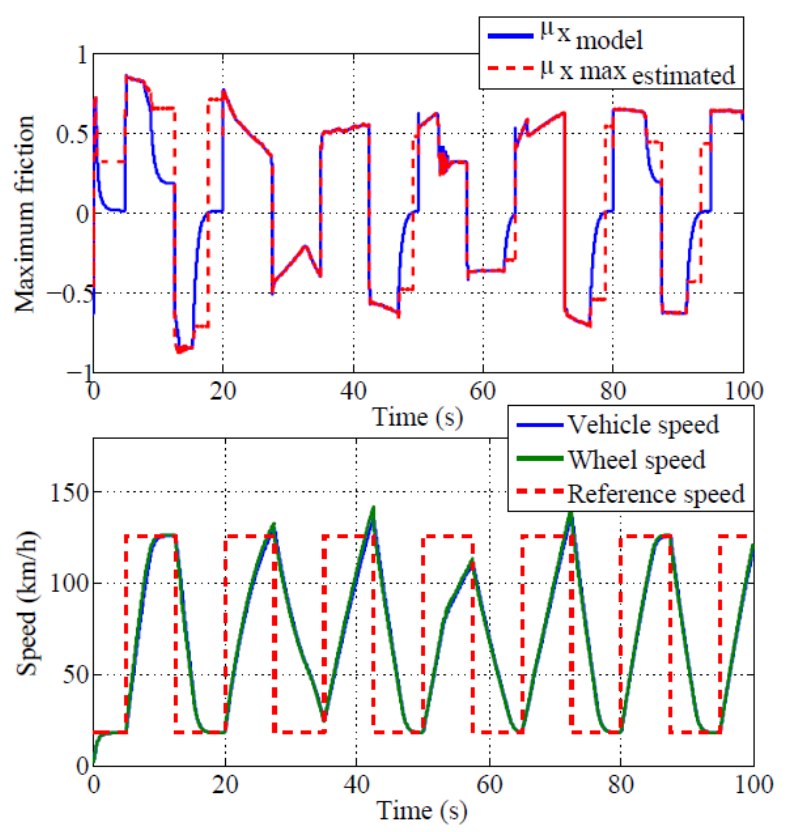

Fig. 16: Speed and maximum friction tracking

As expected, the estimation of $\mu_{x_{\max }}$ gives a larger dispersion of points in noisy environment (figure 17), coming closer to the view seen in real experimental results (see figure 1). This shows that our approach into modeling the road surface conditions comes closer to what is found in real environments. It can be seen in figure 17 that even when the noise affects $\mu_{x_{\max }}$, the parameter $\alpha$ compensates possible estimation errors, therefore, the peak of the curves is never exceeded, showing the robustness of our method.

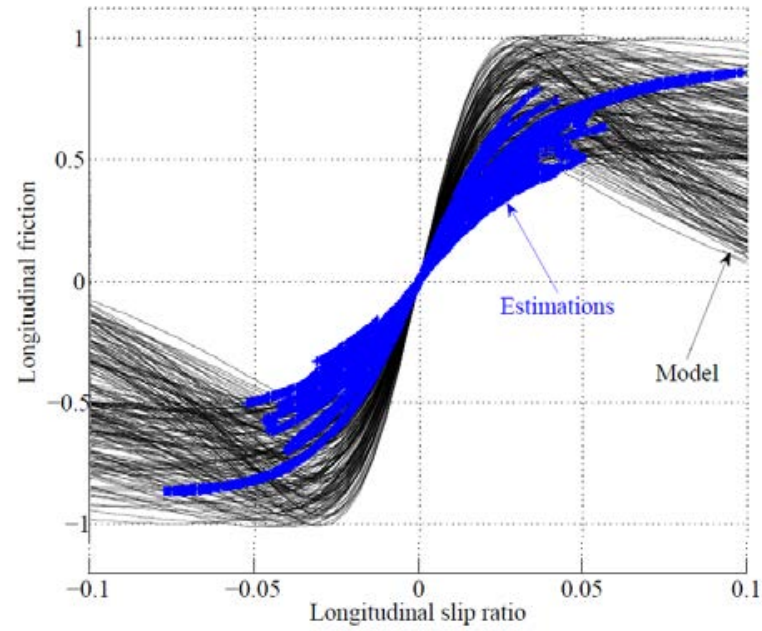

Fig. 17: Maximum friction estimation in noisy environment

A closer look on the points shows the behavior of the estimation and control strategy. In figure 18 can be observed that the transition from one type of road surface to another is made in a continuous manner, as it arrives in actual tire-road environments.

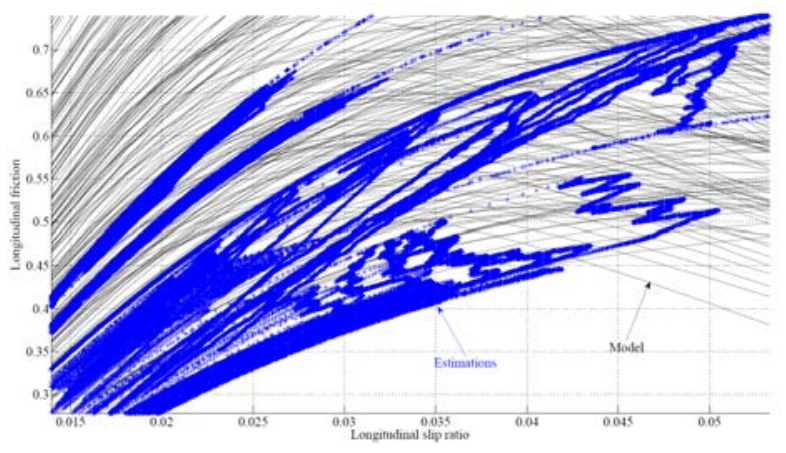

Fig. 18: Maximum friction estimation in noisy environment

The overall estimation process, gives the expected results, detecting the variation of road conditions even when the estimation process is affected by noise. Some of the noise is attenuated by the electric motor [10] and the adaptation parameter $\alpha$, achieving the tracking of the maximum available friction in varying surface conditions.

\section{CONCluding REMARKS}

In this paper, a new approach regarding the modeling of road surface conditions has been presented. It considers a continuous variation of the friction curves, as it arrives in true environments. A "dynamic" behavior of Pacejka parameters is considered in order to model a state of the road variable, $X_{r}$. On this new approach of tire-road model, a maximum friction estimation method based on Dugoff model has been tested in noise-free and noisy conditions, giving promising results in terms of adaptation to operating conditions. Even though the estimation process is computed on a time-varying road conditions, and in addition affected 
by noise, it provides maximum friction values that do not exceed the peak of the friction curves. The results show that the estimation and control strategies perform well in complex circumstances, providing robustness to the proposed method. All the accomplishments were "facilitated" by the use of the electric motor and its consideration as an unique actuator. The electric motor provided the knowledge of the instantaneous torque transmitted at the wheels. In a conventional ICE vehicle, the complexity of the approach increases, since an on-line torque estimator has to be set up and the response times of the actuators in ICE vehicle configuration are greater than in an EV configuration.

\section{ACKNOWLEDGMENT}

The work of the first author (Marcel Stefan Geamanu) is financially supported by the CNRS-IFPEN grant 186-654 (2010-2013).

\section{REFERENCES}

[1] L. Alvarez, X. Claeys, R. Horowitz and J. Yi, "Emergency braking control with an observer-based dynamic tire/road friction model and wheel angular velocity measurement," Vehicle System Dynamics, 39(2): 81-97, 2003

[2] M. Denny, "The dynamics of anti-lock brake systems," European Journal of Physics, 26:1007-1016, 2005.

[3] H. Dugoff, P.S. Fancher, L. Segel " Tire performance characteristics affecting vehicle response to steering and braking control inputs," Technical report, Highway Safety Research Institute, Ann Arbor, MI, 1969.

[4] D.A. Eichfeld and H. Klein, "Anti-lock braking system and vehicle speed estimation using fuzzy logic," Proceedings of the 1st Embedded Computing Conference, Paris, 1996.

[5] R. Freeman, "Robust Slip Control for a Single Wheel," University of California, Santa Barbara, 1995.

[6] M.S. Geamanu, A. Cela, G. LeSolliec, H. Mounier, and S.I Niculescu: Maximum friction estimation and longitudinal control for a full inwheel electric motor vehicle. Proceedings of 12th International Conference on Control, Automation and Systems, Korea, 2012.

[7] M.S. Geamanu, A. Cela, G. LeSolliec, H. Mounier, and S.I Niculescu: Road condition estimation and longitudinal control for electric vehicles. Proceedings of 11th International Conference on Control, Automation and Systems, Korea, 2011.

[8] M. Gerard, W. Pasillas-Lepine., E. de Vries, M. Verhaegen, "Improvements to a five-phase ABS algorithm for experimental validation", Vehicle System Dynamics, 2010.

[9] R. Horowitz, L. Olmos and L. Alvarez, "Adaptive emergency braking control with observer-based dynamic tire-road friction model and underestimation of friction coefficient," Proceedings of the 15th IFAC World Congress. Barcelona, Spain, 2002.

[10] A. Hughes, "Electric motors and drives: fundamentals, types, and applications", Elsevier 2006.

[11] R.N. Jazar, "Vehicle dynamics: theory and applications", Springer 2008.

[12] G. F. Mauer, "A Fuzzy Logic Controller for an ABS Braking System," IEEE Transactions on Fuzzy Systems, Vol. 3, No. 4, 1995, pp. 381-388.

[13] W. Pasillas-Lepine, "Hybrid modeling and limit cycle analysis for a class of five-phase anti-lock brake algorithms," Vehicle System Dynamics, 44(2):173-188, 2006.

[14] W.R. Pasterkamp, H.B. Pacejka, "On line estimation of tire characteristics for vehicle control", JSAE Review, vol 16, p.221 - 226, 1995.

[15] L. R. Raya, D. C. Brandea, J. H. Leverb, "Estimation of net traction for differential-steered wheeled robots," Thayer School of Engineering, Dartmouth College, 8000 Cummings Hall, Hanover, NH 03755 and US Army Cold Regions Research and Engineering Laboratory, United States. 